\title{
Host defence mechanisms against bacterial aggression in periodontal disease: Basic mechanisms
}

\author{
Antonio Bascones-Martínez ${ }^{1}$, Marta Muñoz-Corcuera ${ }^{1}$, Susana Noronha ${ }^{1}$, Paula Mota ${ }^{2}$, Cristina Bascones- \\ Ilundain ${ }^{1}$, Julián Campo-Trapero ${ }^{1}$
}

\author{
${ }^{1}$ School of Dentistry. Madrid Complutense University, Spain \\ ${ }^{2}$ School of Dentistry, Instituto Superior de Ciências da Saúde, Portugal
}

Correspondence:

Departamento Medicina y Cirugía Bucofacial.

Facultad de Odontología

Universidad Complutense de Madrid

Plaza Ramón y Cajal, s/n.

28040 Madrid, Spain

antbasco@odon.ucm.es

Received: 25/01/2009

Accepted: 20/05/2009
Bascones-Martínez A, Muñoz-Corcuera M, Noronha S, Mota P, Bascones-Ilundain C, Campo-Trapero J. Host defence mechanisms against bacterial aggression in periodontal disease: Basic mechanisms. Med Oral Patol Oral Cir Bucal. 2009 Dec 1;14 (12):e680-5.

http://www.medicinaoral.com/medoralfree01/v14i12/medoralv14i12p680.pdf

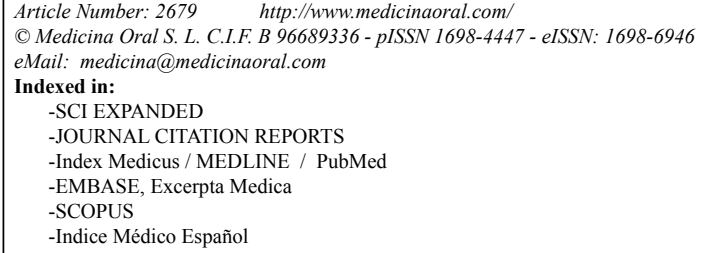

\begin{abstract}
Periodontal diseases are complex bacteria-induced infections characterised by an inflammatory host response to plaque microbiota and their by-products. Most of these microorganisms have virulence factors capable of causing massive tissue destruction both directly, through tissue invasion and the production of harmful substances, or indirectly, by activation of host defense mechanisms, creating an inflammatory infiltrate of potent catabolic activity that can interfere with normal host defense mechanisms. In response to the aggression, host defense mechanisms activate innate and adaptive immune responses. Our aim is to offer a general overview of the main mechanisms involved in the host response to bacterial aggression in periodontitis, such as lipopolysaccharide receptor CD14, complement system, polymorphonuclear neutrophils, antibodies and immunoglobulins.
\end{abstract}

Key words: Periodontitis, humoral response, lipopolysaccharide, neutrophils, CD14.

\section{Introduction}

Periodontitis is a complex infection of bacterial origin in which multiple factors are implicated. It is characterised by an inflammatory host response against microorganisms of the bacterial plaque and their products (1).

Most of these microorganisms can produce tissue destruction $(1,2)$ in two ways: (i) directly, through invasion of the tissues and the production of harmful substances that induce cell death and tissue necrosis; and (ii) indirectly, through activation of inflammatory cells that can produce and release mediators that act on effectors, with potent proinflammatory and catabolic activ- ity. This action plays a crucial role in the destruction of periodontal tissue, while some bacteria also interfere with the normal host defence mechanism by deactivating specific antibodies or inhibiting the action of phagocyte cells (3).

The pathogenesis of periodontal destruction involves the sequential activation of different components of the host immune and inflammatory response, aimed in the first place at defending the tissues against bacterial aggression, reflecting the essentially protective role of the response. However, it also acts as a mediator of this destruction (4). 
The proportion of damage caused by direct effects of the bacteria and that caused by indirect host responsemediated action have yet to be established (3). Although numerous bacteria can degrade tissue directly, Birkedal-Hansen et al (5) suggested that host connective tissue is mainly degraded by the host. Thus, the loss of connective tissue is a defence mechanism; the host attempts self-protection by the apical proliferation of junction epithelium, escaping from the toxic root surface to avoid lesion progression (1-5).

According to the basic principles of epidemiology in infectious diseases, the manifestation of a disease (Fig. 1) depends on interaction between environmental, microbiologic agent- and host-related factors. Thus, specific environmental and genetic factors are likely to determine individual susceptibility for periodontal disease, i.e., for the tissue to be colonised by pathogenic microbiota and infection to develop, giving rise to a destructive inflammatory response (5). The presence of periodontopathogenic bacterial microbiota is a necessary but not sufficient condition for the development of periodontal disease, which can be defined as a mixed infection that causes periodontal destruction in a susceptible host (4).

\section{Host defence mechanisms}

Host defence mechanisms, which interrelate with each other, represent the response of the host to aggression. This response involves the activation of both innate and adaptive elements of the immune system (6).

In periodontitis, bacteria induce an inflammatory response that, alongside their direct destructive effects, is largely responsible for the tissue destruction observed. Inflammatory and immune processes dynamically interact in periodontitis (4), although the great majority of periodontal responses are immunological (1).
Gingival crevicular fluid (GCF) is a serum transudate that originates in the gingival plexus of gingival corium blood vessels, subjacent to the epithelium lining of the dentogingival space. Because it passes through sulcular and junctional epithelia and periodontal tissues, it contains molecular biological markers. If the periodontal inflammation and its severity increase, the GCF augments. GCF contains most of the serum components present in the blood but is enriched with specific components in periodontitis due to the infiltration of inflammatory cells, reflecting the local metabolic state of the periodontal tissues (7-9). Numerous methods for sampling GCF have been developed to date, and over 90 different GCF components have been assessed for periodontal diagnosis (8).

\section{Lipopolysaccharide Receptor: CD 14}

Lipopolysaccharide (LPS) is a virulence factor in the membrane of Gram-negative bacteria membrane, comprising a portion known as lipid $\mathrm{A}$, antigen $\mathrm{O}$ and an oligosaccharide that binds them together. Among these, it is lipid A that can trigger an inflammatory response. LPS is a powerful activator of the innate immune system, which it achieves by stimulating the Toll-like receptor 4 (TLR4), a cell surface protein that recognizes bacterial products $(7,10)$. When bacterial LPS enters the blood circulation, it induces multiple biological responses, including fever, shock, intravascular coagulation, and even death. Some authors have pointed out that serum LPS concentrations rise in periodontitis, increasing the risk of systemic problems (7). The LPS of oral bacteria have a marked effect on most cell types found in periodontal tissues, including macrophages, lymphocytes, fibroblasts and osteoblasts/osteoclasts. Much of the physiopathology of periodontal diseases can be explained by the local biological activity of LPS (7).

\section{Genetic Factors Environmental Factors}

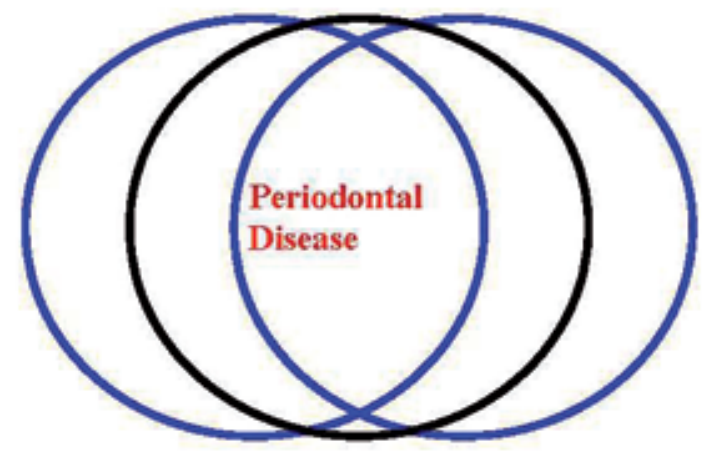

Microbiological Agent

Fig. 1. Factors implicated in periodontal disease. 
LPS binding protein (LBP) is an acute phase reactant, synthesized by hepatocytes, which catalyses LPS transfer to its receptor, membrane CD14 present in monocytes/macrophages or soluble CD14 (sCD14) $(7,10)$ (Fig. 2).

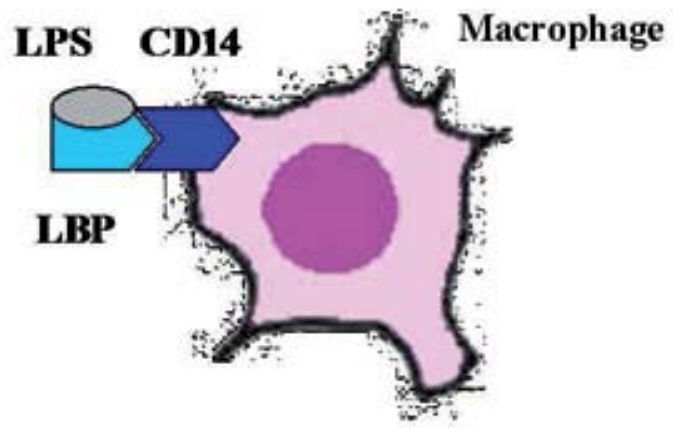

Fig. 2. Binding of lipopolysaccharide (LPS) to its receptor (CD14) prior to binding to LPS binding protein (LBP).

Activation of the CD14 receptor activates monocytes and endothelial cells via a TLR4-dependent pathway, producing the secretion of proinflammatory molecules such as interleukin 1 (IL-1), Tumour Necrosis Factor (TNF), prostaglandin E2 (PG-E2) and interleukin-6 (IL-6). These molecules in turn produce the release of secondary inflammatory mediators, including platelet activation factor (PAF), bioactive aminases (bradykinin and histamine) and prostaglandins $(7,8,10,11)$.

Septic shock exhibits a similar sequence to that observed in periodontal disease: exposure to LPS, release of cytokines and activation of inflammatory mediators (11).

In septic shock, patients with similar LPS values can present different clinical features and cytokine levels, the latter resulting from an alteration in the functioning of the inflammatory response (11). Periodontitis can be considered a disease caused by bacterial microbiota associated with gingivitis in an individual who presents an abnormal inflammatory response.

Hayashi et al. (11) demonstrated the presence of higher CD14 levels in patients with periodontal disease compared with healthy individuals, and suggested that this increase was related to chronic exposure to LPS. Other authors have also demonstrated increase in serum of LPB and antibodies against LPS of periodontopathogens in periodontal patients (7).

\section{Complement system}

The complement system is a group of around 30 proteins that participate in tissue destruction and in inflam- matory processes. It can be activated by the alternative pathway (LPS or other bacterial products) or by the classical pathway (detection of antigen-antibody complexes), giving rise to bacterial opsonisation (2).

Complement activation is considered a protective mechanism in antibacterial immunity, although some products of this pathway may cause tissue destruction. Numerous components of the complement system have been found in the GCF of periodontal patients, either derived from serum or produced by local system activation (9). Many studies have underlined the importance of the complement system in periodontal disease and its role in protecting against periodontopathogenic bacteria (1). Activation of the complement system is one of the earliest host immune responses in the gingival crevicular space (Fig.3). The presence of bacteria in the gingival pocket can activate this system by either the classical or alternative pathway (1). C3 activation varies with the severity of periodontal inflammation, and this can help to differentiate among different forms of periodontitis (9).

Bacteria related to periodontal disease have different mechanisms to evade the complement system $(2,12)$. Some strains present polysaccharides on their surface, which mask the molecules that activate the complement (LPS), increasing the affinity of molecules that inhibit this activity or creating a steric impediment. In other words, hydrocarbon structures on the surface of the bacteria can determine their interaction with components of the complement system (12).

Furthermore, some periodontopathogenic bacteria have proteolytic activity on their cell surface (Fig. 3) that can degrade certain components of the complement system, such as $\mathrm{C} 3$ and $\mathrm{C} 5$, thereby avoiding opsonisation. A further strategy is the release into the medium of molecules that can bind to the complement, reducing the activity of this system on the bacteria (12).

Bacteria such as Porphyromonas gingivalis ( $\mathrm{Pg}$ ) and Actinobacillus actinomycetemcomitans (Aa) have complex hydrocarbon structures with high antigenic and immunogenic potency that can trigger a strong $\operatorname{IgG} 2$ response. IgG2 does not generally fix complement effectively (13).

Cutler et al. (14) demonstrated that the opsonisation and clearance of Pg by neutrophils required the response of specific Ig G-type antibodies. Pg enzyme neutralising antibodies are essential to ensure the normal phagocytic activity of neutrophils when the alternative complement pathway is ineffective. Hence, these antibodies do not function as opsonins but rather serve to neutralise toxic bacterial products, such as proteases.

\section{Polymorphonuclear Neutrophils (PMN)}

Neutrophils are the main cell type in the gingival pocket and form the first line of defence against periodontopathogenic bacteria (Fig. 3) (13). Neutrophil infiltration 


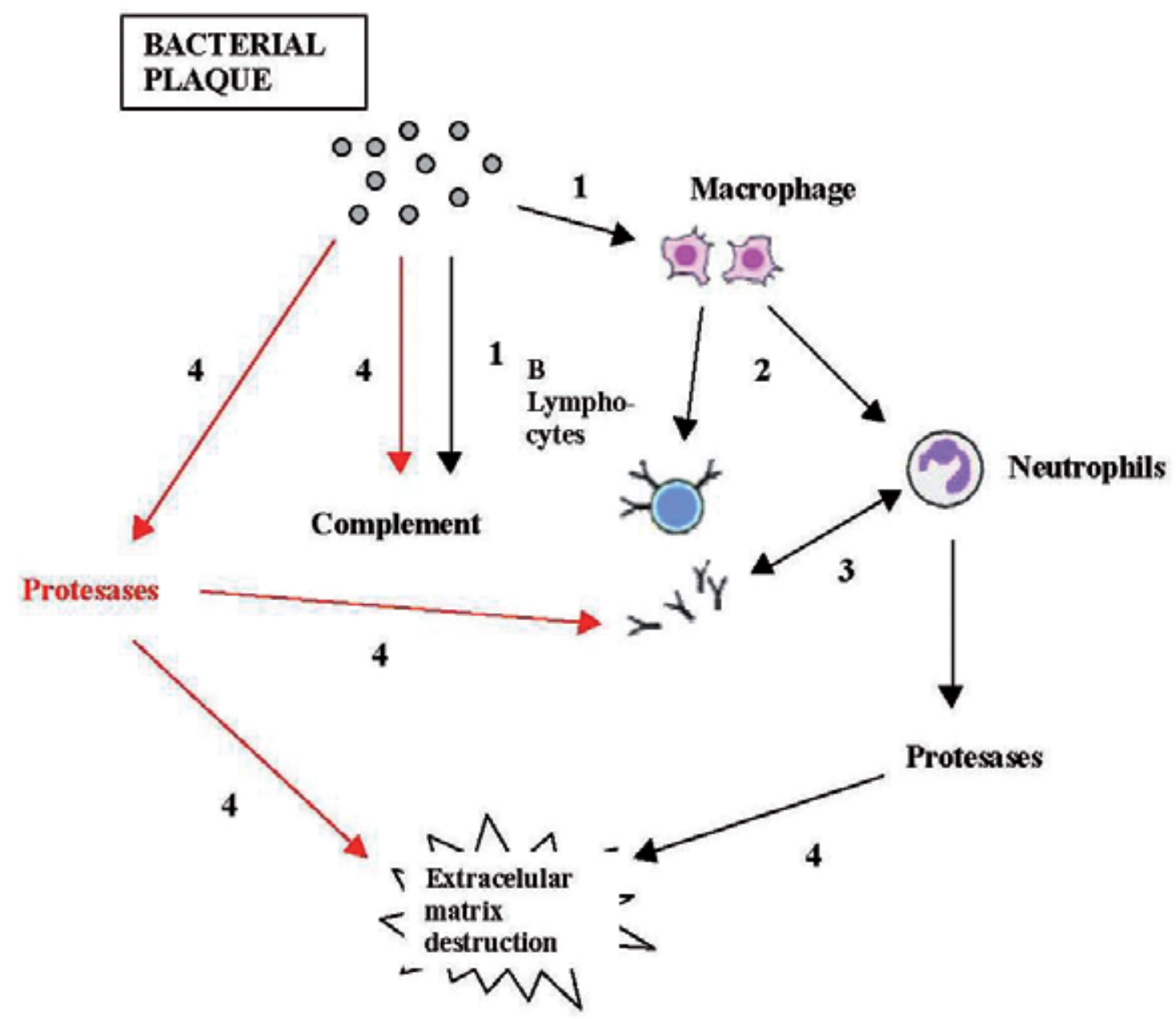

Fig. 3. Some of the mechanisms implicated in the first stages of periodontal disease.

takes place in the periodontal tissue in closest proximity to the colonized root surface (10). Inflammatory cell infiltrate in gingival tissue and GCF is predominantly formed by neutrophils, and B cells and plasma cells are also present (9).Although monocytes are phagocytic cells, they make up the second line of defence and are only activated if the infection persists, when the neutrophils become ineffective (6).

Once in tissue, it is postulated that neutrophils move among cells using the ICAM receptor, following a concentration gradient of IL-8, a chemotactic cytokine for neutrophils $(2,10)$. In the gingival pocket, active neutrophils attempt microbial elimination by phagocytosis. However, some periodontopathogenic bacteria, such as $\mathrm{Pg}$ and $\mathrm{Aa}$, are able to evade the neutrophils, producing a constant flow of these phagocytes into the gingival crevicular space, giving rise to a continuous accumulation and degranulation due to an ineffective phagocytosis $(10,15)$. Degranulation of the PMN is accompanied by the release of endogenous proteases that, alongside the bacterial proteases, produce degradation of the cell matrix. The host proteases are regulated by endogenous inhibitors, which can also modulate the activity of bacterial proteases. Nevertheless, the latter can deactivate the inhibitors, causing continuous destruction of the periodontal tissue (15).

The importance of PMN in periodontal disease is illustrated by the fact that quantitative or qualitative deficiencies in these cells, either congenital (cyclic neutropenia, Chediak-Higashi syndrome, Papillon-Lefèvre syndrome or leukocyte adhesion deficiency) or druginduced (phenylbutazone, penicillin) are associated with a marked destruction of periodontal tissues $(2,6$, 10). Hence, the normal functioning of neutrophils is an essential element of host resistance to periodontal destruction because they form the first protective barrier against inflammation $(10,13)$.

4. Antibodies and Immunoglobulins (Igs)

Immunoglobulins are glycoproteins synthesized by $B$ lymphocytes and plasma cells that have the property 
of specifically binding to the antigen (2). Periodontal pathogens give rise to a marked humoral immune response that can be measured locally in saliva or GCF or systemically in serum (7). Antigen titres vary widely among patients and after treatment of the disease (16). The presence of antibodies against periodontal pathogens in the GCF has been demonstrated in patients with periodontitis (Fig.3), with a predominance of IgG1, with a lesser presence of $\operatorname{IgG} 2$ and an even lower amount of IgG3 and IgG4. IgA1 and IgA2 are found in advanced lesions $(9,16)$. Systemic exposure to pathogens of the oral cavity appears to occur in most patients, inducing the response of specific antibodies (17). The production of antibodies, especially $\operatorname{IgG}$ and $\operatorname{Ig} \mathrm{A}$, is considered to play a protective role against periodontal disease pathogenesis (16). Generally, the highest titles are associated with the most severe forms of the disease. Elevated antibodies to Aa (17) have been reported in localised juvenile periodontitis.

Different antigen-binding models have been described in distinct groups of patients $(18,19)$. These differences in immunoreactivity profile can be related to hydrocarbon molecules, LPS or proteins, although clear immunodominance models have yet to be established for any of the pathogens or patient groups. However, recent studies (20) suggested that the combination of bacterial colonisation and specific antibody responses can effectively discriminate between healthy individuals and those with periodontitis. It has been suggested that antibody responses to a given antigen are regulated by specific genes, so that the response may be more protective for some individuals than for others. Thus, some groups of patients may be more susceptible to periodontitis because they are unable to produce a high-affinity antibody response to the dominant bacterial antigens (21).

The antibody response to specific characteristics of bacterial virulence appears to be critical for the resolution or non-progression of the disease. For example, elevated titres of antibodies against Pg proteases appear in late stages of chronic adult periodontitis and can block proteolytic activity on C3 and Ig G (14).

Ebersole et al. (19) induced periodontitis in monkeys and obtained an elevated serum Ig G and Ig M response in proportion to the increase in the number of microorganisms, supporting the theory that the host antibody response against periodontopathogens is generally protective.

Wheeler et al. (22) found that the severity of the disease was strongly associated with the amount of antibodies and that antibody titre defined the severity with greater accuracy than did level of microorganisms, since transient alterations in serum levels of antibodies against specific bacteria reveal changes in the nature of the infectious load. On the other hand, it should be taken into account that the production of proteolytic enzymes by the microorganisms causes local degradation of Igs in the GCF (Fig. 3).

The type of dominant Ig differs according to the chemical nature of the antigens presented. Thus, protein antigens induce a predominantly IgG1 and IgG4 response, whereas hydrocarbon antigens trigger an $\mathrm{IgG} 2$ response. Genetic factors also play a role, and Afro-Americans have higher serum levels of Aa infection-related $\mathrm{IgG} 2$ in comparison to Caucasians, regardless of periodontal status (23).

Scaling and root planing, fundamental in the treatment of periodontitis, induce a transient systemic bacteremia that re-immunises the patient by increasing the response of serum antibodies to resident oral bacteria. Part of the therapeutic response to scaling and root planning may be attributable to this humoral immunological response, which would increase toxin neutralisation and bacterial clearance (16).

All of these findings suggest a possible role for antibodies as diagnostic indicators and markers of disease activity. However, their value in clinical practice or for patient management has not proven satisfactory. Strictly speaking, elevated levels of antibodies against a periodontal pathogen can only be interpreted as exposure to that pathogen (7).

\section{Final considerations}

Periodontal disease has an infectious aetiology. Its extent and severity depend on the interaction between the bacteria and the host response. The host response to pathogenic microorganisms involves a complex network of cell and humoral components of the immune system, which interact with each other. The progression of gingivitis to periodontitis and the rate of progression of periodontitis cannot be explained solely by the presence of a microbiota. Presence of the bacteria is a necessary but insufficient condition for onset of the disease. Clinical studies have established that individual susceptibility is of great importance in determining disease onset. Hence, an understanding of the factors that influence this susceptibility may be essential to determine periodontal disease activity. These factors include genetic variation between individuals that conditions the appearance of different cell responses. Environmental risk factors, such as bacteria and stress, can alter the balance between host and microbiota, giving rise to the disease. This imbalance may be caused by the proliferation of an exogenous microorganism or the depression of an immune response due to environmental factors. If, on the other hand, the disease results from the excessive growth of endogenous bacteria, a reduction in the host response function can permit this bacterial proliferation.

The accepted model for the progression of periodontitis has changed from the concept of a continuous and 
slow process to a pattern of discontinuous progression characterised by episodes of exacerbation and remission. Thus, onset of the disease requires a pathogen of virulent strain and a host with the necessary genetic factors to trigger the disease. In other words, an individual must be susceptible to a micro-organism and this must be present in sufficient numbers. Other bacterial species must promote or at least not inhibit the process, while the local environment must favour the expression of virulent bacterial properties. If the microorganism is able to evade all of these systems, it triggers the adaptive response, i.e., specific mechanisms.

\section{References}

1. Genco RJ. Host responses in periodontal diseases: current concepts. J Periodontol. 1992;63:338-55.

2. Bascones A, González Moles MA. Mecanismos inmunológicos de las enfermedades periodontales y periimplantarias. Av Periodon Implantol. 2003;15:121-38.

3. Williams RC. Periodontal disease. N Engl J Med. 1990;322:37382.

4. Bascones-Martínez A, Figuero-Ruiz E. Periodontal diseases as bacterial infection. Med Oral Patol Oral Cir Bucal. 2004;9 Suppl:101-7; 92-100.

5. Birkedal-Hansen H. Role of cytokines and inflammatory mediators in tissue destruction. J Periodontal Res. 1993;28:500-10.

6. Kornman KS, Page RC, Tonetti MS. The host response to the microbial challenge in periodontitis: assembling the players. Periodontol 2000. 1997; 14:33-53.

7. Pussinen PJ, Paju S, Mäntylä P, Sorsa T. Serum microbial- and host-derived markers of periodontal diseases: a review. Curr Med Chem. 2007;14:2402-12.

8. Kinney JS, Ramseier CA, Giannobile WV. Oral fluid-based biomarkers of alveolar bone loss in periodontitis. Ann N Y Acad Sci. 2007;1098:230-51.

9. Ebersole JL. Humoral immune responses in gingival crevice fluid: local and systemic implications. Periodontol 2000. 2003;31:135-66.

10. Dixon DR, Bainbridge BW, Darveau RP. Modulation of the in- nate immune response within the periodontium. Periodontol 2000. 2004:35:53-74.

11. Hayashi J, Masaka T, Ishikawa I. Increased levels of soluble CD14 in sera of periodontitis patients. Infect Immun. 1999;67:417-20.

12. Schifferle RE, Wilson ME, Levine MJ, Genco RJ. Activation of serum complement by polysaccharide-containing antigens of Porphyromonas gingivalis. J Periodontal Res. 1993;28:248-54.

13. Haffajee AD, Socransky SS, Taubman MA, Sioson J, Smith DJ. Patterns of antibody response in subjects with periodontitis. Oral Microbiol Immunol. 1995;10:129-37.

14. Cutler CW, Arnold RR, Schenkein HA. Inhibition of C3 and IgG proteolysis enhances phagocytosis of Porphyromonas gingivalis. J Immunol. 1993;151:7016-29.

15. Eick S, Pfister W, Sigusch B, Straube E. Phagocytosis of periodontopathogenic bacteria by crevicular granulocytes is depressed in progressive periodontitis. Infection. 2000;28:301-4.

16. Kinane DF, Lappin DF. Immune processes in periodontal disease: a review. Ann Periodontol. 2002;7:62-71.

17. Shibata K, Warbington ML, Gordon BJ, Kurihara H, Van Dyke TE. Defective calcium influx factor activity in neutrophils from patients with localized juvenile periodontitis. J Periodontol. 2000;71:797-802.

18. Tanner AC, Kent RL Jr, Maiden MF, Macuch PJ, Taubman MA. Serum IgG reactivity to subgingival bacteria in initial periodontitis, gingivitis and healthy subjects. J Clin Periodontol. 2000;27:473-80. 19. Ebersole JL, Cappelli D, Sandoval MN, Steffen MJ. Antigen specificity of serum antibody in A. actinomycetemcomitans-infected periodontitis patients. J Dent Res. 1995;74:658-66.

20. Yeung SC, Taylor BA, Sherson W, Lazarus R, Zhao ZZ, Bird PS, et al. IgG subclass specific antibody response to periodontopathic organisms in HIV-positive patients. J Periodontol. 2002;73:1444-50. 21. Papapanou PN, Neiderud AM, Sandros J, Dahlén G. Checkerboard assessments of serum antibodies to oral microbiota as surrogate markers of clinical periodontal status. J Clin Periodontol. 2001;28:103-6.

22. Wheeler TT, McArthur WP, Magnusson I, Marks RG, Smith J, Sarrett DC, et al. Modeling the relationship between clinical, microbiologic, and immunologic parameters and alveolar bone levels in an elderly population. J Periodontol. 1994;65:68-78.

23. Gunsolley JC, Tew JG, Connor T, Burmeister JA, Schenkein HA. Relationship between race and antibody reactive with periodontitisassociated bacteria. J Periodontal Res. 1991;26:59-63. 